\title{
The role of inter-professional relationships and support for nurse prescribing in acute and chronic pain
}

Karen Stenner BSc (Hons), Research Fellow, School of Health and Social Care, University of Reading, UK. Email: k.l.stenner@reading.ac.uk

Molly Courtenay, MSc, PhD, RN, Professor of Prescribing and Medicines

Management, University of Reading.

This article has been published as: Stenner K., \& Courtenay M. (2008) The role of inter-professional relationships and support for nurse prescribing for patients in acute and chronic pain. Journal of Advanced Nursing, 63 (3), 276-283.

The definite version is available at: www.blackwell-synergy.com 


\begin{abstract}
Aim

This paper is a report of a study exploring nurse prescribers' views on the role of inter-professional relationships and other means of support for nurse prescribing for patients in acute and chronic pain.
\end{abstract}

\title{
Background
}

Research indicates that good team relationships are important for supporting nurse prescribing but that poor understanding of the role by other healthcare professionals can act as a barrier. While collaborative working is central to the role of pain nurses, there is a lack of research on the impact of nurse prescribing on inter-professional working or the support needs of these nurses.

\section{Method}

A qualitative approach was adopted using thematic analysis of semi-structured interview data collected during 2006 and 2007. Participants were 26 nurses who prescribed medicines for patients with acute and/or chronic pain.

\section{Findings}

Nurses' believed that prescribing encouraged collaborative working and sharing of knowledge across professional boundaries and that this helped to broaden understanding of the wider remit of pain management. Collaboration with doctors served a number of functions, including support and continuous learning. Barriers to effective nurse prescribing were a lack of understanding of its role amongst healthcare professionals and inadequate support. Formal support structures, such as regular clinical supervision, were seen as crucial to meeting nurses' ongoing learning.

\section{Conclusion}


Factors that promote understanding of nurse prescribing and support interprofessional relationships are likely to have a positive impact on the effectiveness of nurse prescribing. A more consistent approach is required within organisations to support nurse prescribing.

\section{Keywords}

Nurse prescribing, pain management, support, interprofessional relationships, team working, interviews, empirical research report 


\section{SUMMARY}

\section{What is already known about this topic}

- Inter-professional team-working has been reported to be an important aspect of nurse involvement in treating patients in pain

- Nurse prescribing has the potential to benefit team-working

- Lack of understanding of the nurse prescribing role amongst healthcare professionals can be a barrier to effective nurse prescribing

\section{What this paper adds}

- Inter-professional relationships serve multiple functions for nurses undertaking a prescribing role

- Nurse prescribing for patients in pain encourages sharing of knowledge across boundaries and collaborative working within wider teams

- Access to continuing professional development, supportive clinicians, regular clinical supervision, and peer support networks are crucial to meeting nurses' ongoing learning needs 


\section{INTRODUCTION}

The emphasis placed on the role expansion of nurses (Department of Health (DoH) 1999, DoH 2000) has meant that nurse-led services are seen as one means of improving healthcare provision in the United Kingdom (UK). There is evidence that nurses have lead roles in the delivery of care in a number of treatment areas, including pain (Courtenay \& Carey 2008, Campbell 2004; McKee \& Nolte 2004; Raftery et al. 2005; Courtenay \& Carey 2006). The role of specialist nurses is emphasised for the safe management of acute and chronic pain (Royal College of Anaesthetists and the Pain Society (RCAPS) 2003). Specialist pain nurses have key roles across a variety of settings, including in-patient and out-patient areas in hospitals, in primary care facilities (outreach clinics), oncology and palliative care units within the hospital, and in external sites (RCAPS 2003).

A recent addition to the role of specialist pain nurses in the UK has been the introduction of prescribing, including the ability to prescribe certain Controlled Drugs (CDs) (substances listed in The Misuse of Drugs Regulations 2001), including barbiturates and many opiates. These nurses are able to prescribe as Nurse Independent Prescribers (NIPs) or as Nurse Supplementary Prescribers (NSPs). NIPs are able to assess, diagnose and independently prescribe any licensed medicine (a licence outlines the context in which the medicine can be used), including some CDs, provided that it is within the nurse's area of competence (DoH 2005). By contrast, Nurse Supplementary Prescribing (DoH 2002) takes place after assessment and

diagnosis of a patient's condition have been done by a doctor, and a Clinical Management Plan (CMP) has been drawn up for the patient. The CMP, agreed upon by the patient, nurse (supplementary prescriber) and doctor (independent prescriber), 
includes a list of medicines from which the supplementary prescriber is able to prescribe. NSPs are able to prescribe any medicine (including CDs and unlicensed medicines). This mode of prescribing is best suited to patients with long-term medical conditions or healthcare needs.

Training for NIPs and NSPs is combined. The programme includes 27 days of teaching plus 12 days learning in practice with a medical mentor. Nurses entering the programme must have the ability to study at first degree level and have acquired at least three years' post-registration nursing experience. They must also occupy a post in which they would be required to prescribe, and must be supported by a medical prescriber willing to contribute to the 12 days of learning in practice (Nursing and Midwifery Council (NMC) 2006).

Although several studies have explored the implementation of nurse prescribing, there is a lack of evidence about its impact on nurses working in pain management. Given this dearth of information, a qualitative design was selected to explore the implementation of nurse prescribing from the point of view of nurses working with patients in acute and chronic pain and in palliative care.

\section{BACKGROUND}

Several researchers have attempted to identify the infrastructure necessary to support nurse prescribing (Humphries \& Green 2000, Otway 2002, Courtenay \& Carey 2008). Inter-professional working, which can range from loosely coordinated collaboration to closely organised teamwork within healthcare (Headrick et al 1998), is an important aspect of enabling and supporting nurse prescribing. Peer support, clinical supervision 
and awareness of the role of nurse prescribing by doctors and other healthcare professionals were expected by student nurse prescribers to be necessary to support nurse prescribing, according to findings in a UK study by Humphries and Green (2000). These findings were supported by Otway (2002) in a study involving interviews with 12 UK nurse prescribers and survey data from 241 prescribers. Otway reported that informal peer support, gained through team-working, was an important factor in helping nurse prescribers to develop their professional skills and confidence. She also found that, while support from other healthcare professionals (such as general practitioners) could be valuable, lack of support or understanding of the nurse prescribing role was a barrier.

It was anticipated that nurse prescribing would benefit team relationships by improving communication and enabling more flexible team-working (DoH 1999, 2006). Positive findings with regard to team-working were identified by Bradley and Nolan (2007) in a study using semi-structured interviews to explore the impact of nurse prescribing on a group of 45 recently-qualified NIPs and NSPs. It was evident that the knowledge nurses had gained from a prescribing programme had increased their confidence, enabled them to challenge medication regimes, earned them greater respect from doctors, and helped to increase their networking opportunities with medical prescribers. In addition, respondents reported that other nursing colleagues sought advice from nurse prescribers about medicines and this benefited healthcare teams. However, despite these benefits, there remained a number of misunderstandings about the role and function of nurse prescribing amongst doctors and reception staff. Similar concerns were raised in a study involving focus groups with 46 healthcare professionals in the UK: Hay et al (2004) reported that, while 
teamwork was considered vital to supporting nurse prescribing, there was confusion about the nurse prescribing role and concerns about potential negative affects on team roles. Lack of understanding of the nurse prescriber role by doctors was also reported by participants in a national evaluation of nurse prescribing by Courtenay \& Carey (2008). Respondents reported that lack of understanding acted as a barrier to implementing prescribing. It is therefore evident that teams may have either a positive or a negative influence on nurse prescribing, depending on the level of awareness and understanding of the nurse prescriber role by team members.

While we were unable to identify research specific to nurse prescribing for patients in pain, research about the role of pain nurses in general is available. Nurses were found to play an important role in advising and influencing medical decisions about prescribing for pain in Sweden (Soderhamn \& Idvall 2003) and Canada (Kohr \& Sawhney 2005). In the UK, a questionnaire survey of 103 nurse members of the Pain Society was conducted to explore nurses' beliefs about pain management and their understanding of the roles of members of the pain team (Brown \& Richardson 2006). Interdisciplinary team-working and collaboration were identified as central to the role of nurses in the treatment of pain. Similar findings were reported by Johnston and Smith (2006) in a qualitative study exploring the concept of palliative care and the role of the palliative care nurse. These researchers conducted 44 interviews with patients and nurses in acute and hospice settings in Scotland, and found that a cohesive multidisciplinary team was considered to be a key factor in effective palliative care nursing.

Evidence therefore indicates that inter-professional working is important in pain management and, although not specifically focusing on pain, the success of nurse 
prescribing is influenced by healthcare professionals' understanding and support of the prescribing role. There is no evidence currently available on the views of pain nurses on the adoption of the role of prescribing on their practice. This is an important deficiency, given the emphasis on the role of the nurse prescribing in pain management (Pain Society, 2002).

\section{THE STUDY}

\section{Aim}

The aim of the part of the study reported in this paper was to explore nurse prescribers' views on the role of inter-professional relationships and other means of support for nurse prescribing for patients in acute and chronic pain.

\section{Design}

The data reported here are part of a larger study investigating nurses' views on the adoption of nurse prescribing for pain (Stenner \& Courtenay 2007). A qualitative approach was adopted, using a semi-structured interview technique that allowed researchers to pursue particular issues of relevance as they arose during the interview.

\section{Participants}

A volunteer sample of nurses was recruited from two nationally-recognised pain nurses' groups in England. The 26 participants managed acute, chronic or palliative pain and worked in a variety of clinical roles and work settings. The roles included: clinical nurse specialists in pain $(n=15)$, Macmillan nurses (charity-funded cancer nurses) $(n=2)$, nurse pain consultants $(n=6)$, clinical nurse managers $(n=2)$ and a lead 
pain nurse, i.e. a nurse working in this specialist area and responsible for leading and developing a nursing team. Most participants $(n=19)$ worked in a hospital inpatient and/or outpatient setting and managed acute and/or chronic pain and palliative care, 2 worked in a hospital outpatient department and managed chronic pain and one in a walk-in-centre managing acute and chronic pain. Three participants worked in the community (home care) providing palliative care, and 1 participant managed pain in a hospice. All participants were qualified NIPs/NSPs and worked full-time. Twentyone were female and 5 male.

\section{Data collection}

Details about the study were disseminated to potential participants and those interested in participating were recruited by the group organisers, who then passed contact details of these potential participants to the researchers. We then provided these individuals with further information and answered questions about the project. Interviews were conducted in a quiet location convenient to participants. Each interview was audio-recorded and lasted from 30-50 minutes. Data collection took place between November 2006 and April 2007.

The interview schedule was informed by a review of the literature and national work in the area of nurse prescribing (Courtenay \& Carey 2008, Latter et al 2005). As this paper reports on a subset of data, the interview schedule reflected the broader objectives of the main study (Stenner and Courtenay 2007). Topics covered included general views on nurse prescribing, role changes resulting from prescribing, difficulties in prescribing, enabling factors in prescribing, support and supervision, 
educational needs, views on nurse supplementary prescribing and views on the prescribing course.

\section{Ethical considerations}

The study was approved by the relevant NHS and university ethics committees and service managers. Consent was obtained prior to each interview and participants were free to withdraw from the study at any time. Identifying features were removed from transcripts to anonymise data.

\section{Data analysis}

A thematic analysis was conducted. Variations of this method are widely used in qualitative research. It involves phases of initial familiarisation with the data, coding of transcripts, collating data to identify themes, reviewing and defining themes and producing a written analysis of those themes (Braun and Clarke 2006). The qualitative data analysis software package 'ATLAS' was used to aid initial coding and identification of categories and patterns across the data. A further process of interpreting the broader meanings and importance of the patterns in the data was then used to identify themes. A second researcher independently coded a sample of data and a high level of agreement was reached. Minor differences were resolved through discussion. Saturation was achieved when we were able to code and categorise later interviews without needing to add to the coding framework. The main findings were presented to pain nurses at a pain network meeting in May 2007 and a nurse pain consultant meeting in June 2007. These nurses agreed with and acknowledged the relevance of the findings. 


\section{FINDINGS}

The analysis resulted in five themes: relationships with doctors; peer support and nursing teams; relationships with wider teams; nurses' role in sharing expertise with others; and supportive organisation. The findings are supported by quotations chosen to illustrate either the most typical and commonly reported aspects of each theme, or in some cases, to illustrate variations within a theme.

\section{Relationships with doctors}

Nurses identified building relationships with doctors as important. As a means of support, nurses valued regular contact with at least one clinician in order to discuss clinical issues relevant to their specialist work. If regular clinical supervision sessions were provided, these were an important means of discussing difficult cases, updating, sharing knowledge and building confidence. Arrangements for management and supervision varied from highly structured and regular to irregular and ad hoc. While some nurses had the opportunity to discuss matters with a clinician as they arose, arrangements for formal, regular clinical supervision sessions were not always in place:

We also have clinical supervision every two weeks, so we have got a lot of supervision in place... we meet weekly as a whole team to look at clinical issues, and then weekly as smaller teams with our doctors to go through what we are doing with each of our case loads. 
What I would like to do is set up a more formal system of actual support and development. We did start to have clinical supervision sessions, but they fell by the wayside.

A key feature of developing relationships with general practitioners (GPs) and ward doctors was that nurses regularly communicated their rationales for prescribing decisions to doctors, either orally or in writing. As well as keeping doctors informed, interviewees believed that explaining their rationale would increase doctors' understanding of the decision-making process with regard to prescribing for patients in pain. This was thought to enable sharing of knowledge and expertise, help avoid misunderstandings or disputes, and increase doctors' trust in and willingness to agree with nurse prescribing decisions or recommendations:

I think if you don't work closely with your GPs then yes, it [nurse prescribing] could be negatively impacted. The GP could feel disenfranchised almost and not know what is going on. I have not found that problem. I have a good relationship with my GPs so, if it's complicated or I have done a lot, I will actually make proper telephone contact with them. Occasionally, when that can't happen, I will fax them and say, 'This is what I am doing, please call me if that doesn't make sense.'

I think because we make a point of qualifying what we're prescribing in the notes, then it's all there for people to see, and if people want to challenge it then they can do, but, you know, we can come back at them with a rationale. 
Despite these frequent contacts, not all participants received feedback from doctors (GPs in particular) about prescribing decisions or recommendations. Some GPs only responded when there was a problem, whereas some nurses felt that it would be useful to have constructive feedback on their work as a whole:

We assume things are going well unless we hear otherwise, but that is one of the things - we are actually in the process of meeting and trying to form some type of evaluation, which is likely to be in the form of a questionnaire to the GPs, to really capture in a formal way what they are feeling, if they feel it is useful or not.

\section{Peer support and nursing teams}

Peer support from other nurse prescribers was an important source for gaining information, trouble-shooting and addressing the specific needs of prescribing in pain. It provided encouragement, boosted confidence and helped to build relationships within teams. The level of peer support varied, however. Some participants worked within cohesive teams or met in formal non-medical prescribing groups, others communicated informally, and some were isolated with little peer contact:

We have also set up in this area meeting, and it is predominantly palliative care nurses, just to get together two or three times a year to check out what is going on, what are the issues pertinent to our field, and we always try to have a couple of educational elements to that. It all adds to the confidence. 
Where participants were able to work together as a team, nurse prescribing enabled teams to initiate prescribing decisions immediately and provide more timely and effective care. Actively discussing cases and communicating prescribing rationales within teams also helped nurses to share information and expand their knowledge:

We find, because we work as a team of the acute pain nurses - we're all prescribers - that we follow on from one to the other, and we are very fortunate that my team work very well together. We know each other's work very well. We see the patients the next day and we can titrate it (the medication) to the small amounts.

\section{Relationships with wider teams}

Patients with complex pain required nurses to collaborate with wider interprofessional teams, such as other pain teams, consultants, psychologists, pharmacists and complementary therapists. Interviewees also collaborated with drug treatment centres and mental health services for some patients. Inter-professional relationships were important for sharing advice and information relevant to prescribing decisions. Pharmacists were particularly valued as a source of information and advice on CDs and drug interactions. Measures by pharmacists to double-check prescriptions for accuracy and error were seen as necessary and useful. Gaining a broader understanding of issues affecting prescribing for pain was part of ongoing continual learning for nurses:

A lot of our patients are complex. They shouldn't always have unilateral decisionmaking. (At) the Wednesday morning meeting there is a whole MDT 
[multidisciplinary team], so there is the (medical) consultant, the psychologists and... mostly it is just a discussion between me and one of the doctors and two heads are better than one, really. It's better with a complicated problem to have other people's opinions too.

We have got a pharmacist technician that is absolutely fantastic when it comes to the legislation, particularly around Controlled Drugs, and also the pharmacist is quite helpful in offering advice and being there when we do have queries.

\section{Nurses' role in sharing expertise with others}

Interviewees believed they had an important role to play in sharing their expertise with other healthcare professionals. The daily process of communicating prescribing decisions and recording patient notes formed the basis of this educational role, although some also gave formal education sessions. By doing this, they helped to maintain a level of consistency in evidence-based prescribing within their area of work, which was important where there was a high turn-over of junior doctors on a ward. This educational role was seen as key to avoiding potential deskilling of doctors and nurses previously involved in tasks now conducted by nurse prescribers, which was a particular concern in hospital settings. Some nurses worried that increasing workloads could diminish their educational role and ultimately reduce the knowledge and skill level of junior doctors:

One of my concerns was if I just 'prescribe and go' - or if all nurses just 'prescribe and go' - where are doctors going to get their continued professional development from? Because we know there is less senior medical support on the 
wards, so you have got new doctors coming out who were given information by nurses before, who may now have specialists [nurses] just wandering around prescribing, particularly on the acute pain wards (wards where patients were experiencing pain for acute conditions).

Participants were also involved in explaining the nurse prescribing role to healthcare professionals. Misunderstandings, such as where nurses were asked to prescribe beyond their area of competence or for patients whom they had not directly assessed, were commonly reported. Although rare, there were instances where the ability to make full use of the potential of nurse prescribing had been hampered by misunderstanding or resistance by other professionals. Informing colleagues that a nurse will be prescribing and giving details of the areas in which they will prescribe seemed to help smooth potential difficulties:

We only had one issue with one GP in a practice and he does not want us to prescribe for his patients at all. He does not want us stopping drugs at all, not even things like statins for cholesterol when the patient is in their terminal phase. That is a barrier to our managing the patients.

\section{Supportive organisation}

Alongside inter-professional support, a number of organizational factors were considered important for supporting nurse prescribing. These included clear local policy and guidance on prescribing, access to continuing professional development (CPD), formal support and a learning environment that encouraged sharing knowledge across professional boundaries. 
Nurses' own involvement in developing local policy and protocol for non-medical prescribing was an important aspect for those working at consultant or managerial level. Four participants represented non-medical prescribers on Drugs and Therapeutic Committees, and other involvement included conducting audit and developing guidelines, education and protocols for prescribing. Recognition of nonmedical prescribing at a high level in a healthcare organisation was considered important in encouraging such involvement by nurse prescribers:

My trust (provider organisation) has been very supportive of non-medical prescribers and they felt it very important that a non-medical prescriber actually sat on the Drugs and Therapeutics Committee... because they've got somebody that is actually on the ground floor.

There was, however, some debate over the extent to which nurse prescribing should be regulated within organizations. On the one hand, local formulary restrictions and formal agreements (such as an 'intent to prescribe'), were helpful in defining the limits of practice and assisting nurses to resist pressure from patients or professionals to prescribe outside of their area of competence. On the other hand, local policy threatened to limit nurses if it was too stringently defined. A balance was required, with policy general enough to support nurse prescribing without being too restricting:

We don't want to over-protocolise it. Once you have qualified and you know your competencies and you work within the NMC (Nursing \& Midwifery Council - the UK regulating authority) code of conduct, then as long as you 
keep us informed of your prescribing areas and practices and you can assure us you are working within that loose guideline, then get on with it, really.

Participants used a variety of resources to keep up-to-date with clinical developments. Many were involved with local and national networks and regularly attended meetings and conferences. Other resources used were journals, the internet, the British National Formulary (BNF) and support provided by pharmaceutical drug representatives. However, interviewees did experience problems accessing CPD, mainly due to lack of funding and protected learning time to release staff. Changes to local structures and job losses had resulted in lack of managerial support and organization for CPD specific to nurse prescribing, and participants had had problems in accessing adequate support at an appropriate level:

Because of the financial situation of the trust, actually getting funding for and getting time to attend courses at the moment is very difficult.

I think the problem with that is, as with many of the things that have come up against us as a nurse consultant, is that there isn't really anybody around to give me the support. You know, I am the first one to start prescribing, so arguably there is no one else in my hospital with more experience as a nurse prescriber. It is actually very difficult to find mentorship and clinical supervision that is actually really, really relevant.

\section{DISCUSSION}


This was a small explorative study for which a qualitative design was deemed appropriate, given that little is known about how nurse prescribing affects interprofessional relationships in this context. The use of thematic analysis gave an overview of commonly-occurring themes that may be used to inform future research. Participants were mainly pain specialists, most of whom worked in hospital settings. Further research on the role of generalist nurse prescribing for patients in pain in primary care settings is needed. Although our findings provide evidence that interprofessional relationships are of central importance to nurse prescribing for pain, further research is needed to explore the views of doctors, pharmacists and patients.

Our findings show that nurses' relationships with doctors serve multiple functions. Contact with clinicians who have expertise in treating patients in pain was vital for gaining support and enabling continuous learning and development. This is consistent with an observation by Fairly (2006) that constant exposure to clinical events under the direction and tutorship of an experienced clinician is essential for developing expert clinical and diagnostic skills. In addition, nurses create opportunities to share their specialist knowledge with doctors through communicating their prescribing rationales. Although it is a requirement for nurse prescribers to record prescription details in patients' records (DoH 2006), these nurses were providing further information, particularly about complex or unusual prescriptions. This served an educational function that was believed to counterbalance the potential effect of deskilling that might occur through having specialist nurses prescribing for patients in pain, particularly in hospital wards. In other words, nurses believed that they were instrumental in maintaining a good level of knowledge and skill amongst healthcare professionals and helped to achieve greater consistency in prescribing for pain. 
Communicating rationales further served to avoid disputes with doctors and helped to increase doctors' trust in nurses' prescribing decisions. While direct challenges to nurses' prescribing decisions were rare, resistance to nurse prescribing by doctors did occur, as has been reported elsewhere (Otway 2002, Wilhelmson and Foldevi 2003). Interestingly, nurses believed that the process of developing trusting relationships with doctors helped to reduce the number of challenges to their decisions, although they seldom received any direct feedback from GPs about this.

Nurses are only able to prescribe medicines within their own areas of competence (NMC 2006). In our study, interviewees reported that their depth of experience enabled them to define their area of competence; however, there was general agreement that misunderstandings existed about nurse prescribing. Educating other members of the healthcare team about this was seen by interviewees as a way by which this problem might be overcome. Policies requiring nurses explicitly to record their areas of competence were helpful, although restrictive if too stringent. The need for healthcare professionals to understand this aspect of the prescribing role is therefore emphasised by our findings and supports findings by Humphries \& Green (2000) and Otway (2002) that understanding of the role of the nurse prescriber by the medical profession is necessary if nurse prescribing is to be effective.

Consistent with findings from studies on pain nursing in general, inter-professional and collaborative working was an important aspect of prescribing for patients in pain (Brown and Richardson 2006, Johnston and Smith 2006). Participants frequently liaised with a number of different professionals, particularly with regard to complex 
patients. This enabled sharing of knowledge across boundaries and gave opportunities for nurses to broaden their understanding of pain management. Similarly, access to peer support was an important means of developing confidence and sharing knowledge about prescribing, as has been reported by previous researchers (Humphries and Green 2000, Otway 2002). There are indications that sharing knowledge in this way benefits the wider team as well as the nurse prescriber (Bradley and Nolan 2007). Jones et al (2007), in a recent study of mental health nurse supplementary prescribing, reported that the existence of a nurse prescribing team had a positive effect on the knowledge, skills and practice of the entire mental health team.

Our participants made use of a variety of resources for support and development. Alongside support from doctors, pharmacists, wider teams and peers, they also accessed written material, conferences and networks for information and support. Community nurse prescribers in the UK have been reported to use a similar range of information sources (Hall et al, 2003). However, access to formal support, whilst recognised as important by interviewees, was inconsistent. Inadequacies in the provision of clinical supervision and access to CPD have been consistently reported in studies of nurse prescribing (Humphries \& Green 2000, Otway 2002, Luker and McHugh 2002, Courtenay et al 2007, Jones et al 2007). Nurses in Otway's (2002) study reported that, in the absence of such support, they found alternative methods of keeping up-to-date, such as peer support networks. Although our participants were able to draw on a wide range of resources, some worked in organisations with a poor supportive infrastructure, were unable to access CPD and were isolated from other means of support, such as peer support networks. This is important, as nurses working 
in isolation with poor peer support networks have been found to lack confidence and prescribe less than those enjoying the benefits of support (Otway 2002).

\section{CONCLUSION}

The implication of this study is that good interprofessional relationships with doctors, peers and wider teams are essential for supporting effective nurse prescribing for pain. These relationships are an important means for both sharing and gaining knowledge and expertise. It is important to acknowledge the role that nurses themselves can play in actively encouraging understanding about nurse prescribing and fostering good relationships through their educational role with other professionals. Greater attention should be paid to providing adequate infrastructure within organisations to support nurse prescribing. 


\section{REFERENCES}

Bradley E., \& Nolan P. (2007) Impact of nurse prescribing: A qualitative study. Journal of Advanced Nursing 59 (2), 120-128.

Braun V. \& Clarke V. (2006) Using thematic analysis in psychology. Qualitative Research in Psychology 3, 77-101.

Brown C. A \& Richardson C. (2006) Nurses' in the multi-professional pain team: A study of attitudes, beliefs and treatment endorsements. European Journal of Pain 10, 13-22.

Campbell N. (2004) Secondary Prevention clinics: improving quality of life and Outcome. Heart 90, (Suppl iv), 29-32.

Courtenay M. \& Carey N. (2006) Nurse-led care in dermatology: A review of the literature. British Journal of Dermatology 154, 1-6.

Courtenay M, Carey N. (2008). Nurse Independent Prescribing and Nurse Supplementary Prescribing: Findings from a national questionnaire survey. Journal of Advanced Nursing, 61 (3), 291-299

Courtenay, M \& Carey, N.J. (2008). The impact and effectiveness of nurse-led care in the management of acute and chronic pain. Journal of Clinical Nursing (accepted for publication)

Department of Health (1999) Making a Difference. Strengthening the Nursing, Midwifery and Health Visiting Contribution to Healthcare. Department of Health, London

Department of Health (2000) The NHS Plan: A Plan for Investment, A plan for reform. Department of Health, London.

Department of Health (2002).Supplementary prescribing. Department of Health, London. 
Department of Health (2005) Written Ministerial Statement on the expansion of independent nurse prescribing and introduction of pharmacists independent prescribing, Department of Health, London.

Department of Health (2006). Independent prescribing by Nurses and Pharmacists within the NHS in England. A Guide for Implementation. Department of Health, London

Fairley D (2006) Factors influencing effective independent nurse prescribing. Nursing Times 102 (31), 34-37.

Hay A., Bradley E. \& Nolan P. (2004) Supplementary nurse prescribing. Nursing Standard 18 (41), 33-39.

Hall J., Cantrill J. \& Noyce P. (2003) Professional issues. The information sources used by community nurse prescribers. British Journal of Nursing 12 (13), 810818.

Headrick LA, Wilcock PM \& Batalden PB. (1998) Interprofessional Working and Continuing Professional Education. British Medical Journal 316, 771-774.

Humphries J.L. \& Green E. (2000) Nurse prescribers: infrastructures required to support their role. Nursing Standard 14 (48), 35-39.

Johnston B. \& Smith L.N. (2006) Nurses' and patients' perceptions of expert palliative care. Journal of Advanced Nursing 54 (6), 700-709.

Jones M., Bennett J., Lucas B., Miller D. \& Gray R (2007) Mental health nurse supplementary prescribing: experiences of mental health nurses, psychiatrists and patients. Journal of Advanced Nursing 59 (5), 488-496.

Kohr R. \& Sawhney M. (2005) Advanced Practice Nurses' Role in the Treatment of Pain, Canadian Nurse 101 (3), 30-34. 
Latter S., Maben J., Myall M., Courtenay M., Young, A. \& Dunn N. (2005) An evaluation of extended formulary independent nurse prescribing. Executive summary of final report. Department of Health, London.

Luker K.A \& McHugh G. (2002) Nurse prescribing from the community nurse's perspective. The International Journal of Pharmacy Practice 10, 273-280.

McKee M. \& Nolte E. (2004) Responding to the challenge of chronic diseases: ideas from Europe. Clinical Medicine 4, 336-42.

NMC (2006) Standards of Proficiency for nurse and midwife prescribers. NMC, London.

Otway C. (2002) The development needs of nurse prescribers. Nursing Standard 16 (18), 33-38.

Pain Society (2002) Recommendations for Nursing Practice in Pain Management. Pain Society, London.

Raftery J.P., Yao G.L., Murchie P., Campbell N.C. \& Ritchie L.D. (2005) Cost effectiveness of nurse led secondary prevention clinics for coronary heart disease in primary care: follow up of a randomised controlled trial. $B M J \mathbf{3 3 0}$ (7493), 707-711.

RCAPS (2003) Pain Management Services: Good Practice. The Royal College of Anaesthetists \& The Pain Society, 1-22 May, London.

Söderhamn O. \& Idvall E. (2003) Nurses' influence on quality of care in postoperative pain management: A phenomenologcial study. International Journal of Nursing Practice 9, 26-32.

Stenner K. \& Courtenay M. (2007). A qualitative study on the impact of legislation on the prescribing of controlled drugs by nurses. Nurse Prescribing 5 (6), 257261. 
Wilhelmsson S. \& Foldevi M (2003) Exploring views on Swedish district nurses' prescribing - a focus group study in primary health care. Journal of Clinical Nursing 12, 643-650. 